\title{
On the Statistical Independence of Compound Pseudorandom Numbers Over Part of the Period
}

\author{
MORDECHAY B. LEVIN \\ Bar-Ilan University
}

\begin{abstract}
This article deals with the compound methods with modulus $m$ for generating uniform pseudorandom numbers, which have been introduced recently. Equidistribution and statistical independence properties of the generated sequences over part of the period are studied based on the discrepancy of $d$-tuples of successive pseudorandom numbers. It is shown that there exist parameters in compound methods such that the discrepancy over part of the period of the corresponding point sets in the $d$-dimensional unit cube is of an order magnitude of $O\left(N^{-1 / 2}(\log N)^{d+3}\right)$ for all $N=1, \ldots, m$. This result is applied to the compound nonlinear, inversive and explicit inversive congruential methods.
\end{abstract}

Categories and Subject Descriptors: G.3. [Probability and Statistics]: random number generation; I.6. [Simulation and Modeling]: General

General Terms: Theory

Additional Key Words and Phrases: Diaphony, discrepancy, theoretical tests

\section{INTRODUCTION}

Equidistribution and statistical independence properties of uniform pseudorandom numbers can be analyzed based on the discrepancy of certain point sets in $[0,1)^{d}$. For $N$ arbitrary points, $\mathbf{t}_{0}, \mathbf{t}_{1}, \ldots, \mathbf{t}_{N-1} \in[0,1)^{d}$, the discrepancy is defined by

$$
D\left(\mathbf{t}_{0}, \ldots, \mathbf{t}_{N-1}\right)=D\left(\left(\mathbf{t}_{n}\right)_{n=0}^{N-1}\right)=\sup _{J}\left|F_{N}(J)-V(J)\right|,
$$

where the supremum is extended over all subintervals $J$ of $[0,1)^{d}, F_{N}(J)$ is $N^{-1}$ times the number of points among $\mathbf{t}_{0}, \mathbf{t}_{1}, \ldots, \mathbf{t}_{N-1}$ falling into $J$, and $V(J)$ denotes the $d$-dimensional volume of $J$.

Let us consider pseudorandom numbers obtained by means of the linear congruential method

$$
x_{1}(n)=\left\{\frac{y_{n}}{p}\right\}, \quad y_{n+1} \equiv \lambda y_{n}(\bmod p), \quad y_{0}=c_{0}, \quad n=0,1, \ldots
$$

This work was supported in part by the Israel Science Foundation Grant No. 366-172.

Author's address: Department of Mathematics and Computer Science, Bar-Ilan University, 52900, Ramat-Gan, Israel, e-mail: mlevin@macs.biu.ac.il.

Permission to make digital/hard copy of part or all of this work for personal or classroom use is granted without fee provided that the copies are not made or distributed for profit or commercial advantage, the copyright notice, the title of the publication, and its date appear, and notice is given that copying is by permission of the ACM, Inc. To copy otherwise, to republish, to post on servers, or to redistribute to lists, requires prior specific permission and/or a fee.

(C) 2001 ACM 1049-3301/01/0700-0294 \$5.00 
and shift-register methods

$$
\begin{aligned}
x_{2}(n) & =\sum_{i=1}^{k} \frac{z_{k n+i-1}}{p^{i}}, \quad x_{3}(n)=\sum_{i=1}^{k} \frac{z_{n+d_{i}}}{p^{i}}, \\
z_{n+k} & \equiv a_{k} z_{n+k-1}+\cdots+a_{1} z_{n}(\bmod p), \\
z_{0} & =c_{1}, \ldots, z_{k-1}=c_{k}, \quad n=0,1, \ldots,
\end{aligned}
$$

where $p$ is prime and $f(x)=x^{k}-a_{k} x^{k-1}-\cdots-a_{1}$ is the characteristic polynomial of the sequence $\left(z_{n}\right)_{n \geq 0}$.

In a series of papers, Niederreiter (see the reviews in Niederreiter [1977; $1978 ; 1992]$ ) proved that there exists a multiplier $\lambda$ (a characteristic polynomial $f(x)$ ) such that for $i=1,2,3$

$D\left(\left(x_{i}(n), \ldots, x_{i}(n+d-1)\right)_{n=0}^{N-1}\right)=O\left(\frac{\sqrt{m}(\log m)^{d+1}}{N}\right), \quad$ for $\quad N=1, \ldots, m$,

where $m$ is the period of the sequence of pseudorandom numbers.

This estimate is interesting for $N \gg \sqrt{m}(\log m)^{d+1}$. According to Levin [1989], we can obtain a nontrivial discrepancy estimate also for a small part of the period: there exists a multiplier $\lambda$, a characteristic polynomial $f(x)$ and initial values $c_{0}, c_{1}, \ldots, c_{k}$ such that for $i=1,2,3$

$D\left(\left(x_{i}(n), \ldots, x_{i}(n+d-1)\right)_{n=0}^{N-1}\right)=O\left(N^{-1 / 2}(\log N)^{d+3}\right), \quad$ for $\quad N=1, \ldots, m$.

The smallest previously known discrepancy estimate over part of the period for other pseudorandom number generators satisfies

$$
D\left((u(n), \ldots, u(n+d-1))_{n=0}^{N-1}\right)=O\left(\frac{\sqrt{m}(\log m)^{d+1}}{N}\right), \quad \text { for } \quad N=1, \ldots, m,
$$

where $m$ is the period of the sequence $\left(u_{n}\right)_{n \geq 0}$ (see the reviews and references in Eichenauer-Herrmann [1995] and Niederreiter [1992]).

Our goal is to obtain a nontrivial discrepancy estimate for a small part of the period for nonlinear pseudorandom numbers generators. In this article, we obtain a result similar to (1) for compound pseudorandom number generators that have been introduced recently (see the reviews in Drmota and Tichy [1997], Eichenauer-Herrmann [1995] and L'Ecuyer [1994]).

Let $p_{1}, \ldots, p_{r}$ be arbitrary distinct primes. For $1 \leq i \leq r$, identify the set $\mathbb{Z}_{p_{i}}=\left\{0,1, \ldots, p_{i}-1\right\}$ with the finite field of order $p_{i}$, and let $\mathbb{Z}_{p_{i}}^{*}=\mathbb{Z}_{p_{i}} \backslash\{0\}$ be its multiplicative group. Let $\left(\mathbf{z}_{n}^{(i)}\right)_{n \geq 0}$ be a sequence of elements of $\mathbb{Z}_{p_{i}}^{d}$, which is purely periodic with period length $p_{i}$. For a parameter $c_{i} \in \mathbb{Z}_{p_{i}}^{*}$, let $\left(\mathbf{x}_{n}^{(i)}\right)_{n \geq 0}$ with

$$
\mathbf{x}_{n}^{(i)} \equiv \frac{c_{i} \mathbf{z}_{n}^{(i)}(\bmod 1)}{p_{i}}, \quad n \geq 0
$$

be the corresponding stream of $d$-dimensional vectors of (ordinary) pseudorandom numbers in the interval $[0,1)$. A sequence $\left(\mathbf{x}_{n}\right)_{n \geq 0}$ of $d$-dimensional vectors of compound pseudorandom numbers in the interval $[0,1)$ is defined by

$$
\mathbf{x}_{n} \equiv \mathbf{x}_{n}^{(1)}+\cdots+\mathbf{x}_{n}^{(r)}(\bmod 1), \quad n \geq 0 .
$$


Since the primes $p_{1}, \ldots, p_{r}$ are distinct, it follows by standard arguments that the sequence $\left(\mathbf{x}_{n}\right)_{n \geq 0}$ is purely periodic with period length $m=p_{1} \cdots p_{r}$. Compound methods are particularly suitable for parallelized computations, since the underlying sequences $\left(\mathbf{x}_{n}^{(i)}\right)_{n \geq 0}$ can be computed by parallel processors.

In the following, the $d$-dimensional vectors $\mathbf{x}_{0}, \mathbf{x}_{1}, \ldots, \mathbf{x}_{m-1}$ of compound pseudorandom numbers are considered and the abbreviation

$$
D_{N ; c_{1}, \ldots, c_{r}}^{(d)}=D\left(\mathbf{x}_{0}, \mathbf{x}_{1}, \ldots, \mathbf{x}_{N-1}\right)
$$

is used for $1 \leq N \leq m=p_{1} \cdots p_{r}$.

In the third section, we show that there exist parameters $\left(c_{1}, \ldots, c_{r}\right) \in \mathbb{Z}_{p_{1}}^{*} \times$ $\cdots \times \mathbb{Z}_{p_{r}}^{*}$ such that

$$
D_{N ; c_{1}, \ldots, c_{r}}^{(d)}=O\left(N^{-1 / 2}(\log N)^{d+3}\right), \quad \text { for } \quad N=1, \ldots, m,
$$

where the $O$-constant depends on the number of points among $\mathbf{z}_{0}^{(i)}, \mathbf{z}_{1}^{(i)}, \ldots, \mathbf{z}_{p_{i}-1}^{(i)}$ falling into certain hyperplanes over the finite field $\mathbb{Z}_{p_{i}}(i=1, \ldots, r)$. To prove this we use the approach proposed in Levin [1989] and the average discrepancy estimate obtained in Eichenauer-Herrmann et al. [1997]. A similar result is obtained also for the other characteristic of equidistribution and statistical independence properties of uniform pseudorandom numbers, for the diaphony (see Drmota and Tichy [1997], Hellekalek and Niederreiter [1998], and Zinterhof [1976]). A heuristic algorithm of choosing parameters $\left(c_{1}, \ldots, c_{r}\right)$ is proposed in this section. In the fourth section, the assertion (5) is applied to the compound nonlinear, inversive and explicit inversive congruential method. The second section contains necessary auxiliary results.

\section{AUXILIARY RESULTS}

First, some further notation is necessary. For integers $d \geq 1$ and $q \geq 2$, let $C_{d}(q)$ be the set of all nonzero lattice points $\left(h_{1}, \ldots, h_{d}\right) \in \mathbb{Z}^{d}$ with $-q / 2<h_{j} \leq q / 2$ for $1 \leq j \leq d$. Define

$$
r(h, q)=\left\{\begin{array}{cl}
q \sin (\pi|h| / q) & \text { for } \quad h \in C_{1}(q) \\
1 & \text { for } h=0
\end{array}\right.
$$

and

$$
r(\mathbf{h}, q)=\prod_{j=1}^{d} r\left(h_{j}, q\right)
$$

for $\mathbf{h}=\left(h_{1}, \ldots, h_{d}\right) \in C_{d}(q)$. For real $t$, the abbreviation $e(t)=e^{2 \pi \sqrt{ }-1 t}$ is used and $\mathbf{u} \cdot \mathbf{v}$ stands for the standard inner product of $\mathbf{u}, \mathbf{v} \in \mathbb{R}^{d}$. Subsequently, three known results are stated, which follow from Niederreiter [1992, Theorem 3.10 and p. 35], Korobov [1992, p. 13], Eichenauer-Herrmann et al. [1997, Lemma 3], and Niederreiter [1977, Lemma 2.3], respectively.

Lemma 1. Let $N \geq 1$ and $q \geq 2$ be integers. Let $\mathbf{t}_{n}=\mathbf{y}_{n} / q \in[0,1)^{d}$ with $\mathbf{y}_{n} \in\{0,1, \ldots, q-1\}^{\bar{d}}$ for $0 \leq n<N$. Then the discrepancy of the points 
$\mathbf{t}_{0}, \mathbf{t}_{1}, \ldots, \mathbf{t}_{N-1}$ satisfies

$$
D\left(\mathbf{t}_{0}, \mathbf{t}_{1}, \ldots, \mathbf{t}_{N-1}\right) \leq \frac{d}{q}+\frac{1}{N} \sum_{\mathbf{h} \in C_{d}(q)} \frac{1}{r(\mathbf{h}, q)}\left|\sum_{n=0}^{N-1} e\left(\mathbf{h} \cdot \mathbf{t}_{n}\right)\right| .
$$

Lemma 2. Let $T \geq N \geq 1$ be integers. Then

$$
\left|\sum_{n=0}^{N-1} e\left(t_{n}\right)\right| \leq \sum_{h_{0} \in(-T / 2, T / 2]} \frac{1}{r\left(h_{0}, T\right)}\left|\sum_{n=1}^{T} e\left(t_{n}+\frac{n h_{0}}{T}\right)\right| .
$$

PRoof. According to Niederreiter [1992, p. 35], 1/T| $\sum_{n \in[0, N)} e(n h / T) \mid \leq$ $r^{-1}(h, T)$. Now, repeating the proof of Korobov [1992, p. 13], we obtain the assertion of Lemma 2.

Corollary 1. Let $T \geq N \geq 1$ and $q \geq 2$ be integers, $\mathbf{t}_{n}=\mathbf{y}_{n} / q \in[0,1)^{d}$ with $\mathbf{y}_{n} \in\{0,1, \ldots, q-1\}^{d}$ for $0 \leq n<N$. Then

$$
D\left(\mathbf{t}_{0}, \mathbf{t}_{1}, \ldots, \mathbf{t}_{N-1}\right) \leq \frac{d}{q}+\frac{T}{N} \tilde{D}\left(\left(\mathbf{t}_{n}\right)_{n=0}^{T-1}\right),
$$

where

$$
\tilde{D}\left(\left(\mathbf{t}_{n}\right)_{n=0}^{T-1}\right)=\frac{1}{T} \sum_{\mathbf{h} \in C_{d}(q)} \sum_{h_{0} \in(-T / 2, T / 2]} \frac{1}{r(\mathbf{h}, q) r\left(h_{0}, T\right)}\left|\sum_{n=0}^{T-1} e\left(\mathbf{h} \cdot \mathbf{t}_{n}+\frac{n h_{0}}{T}\right)\right| .
$$

Lemma 3 . Let $q \geq 2, T \geq 1$ be integers. Then

$$
\sum_{\substack{\mathbf{h} \in C_{d}(q) \\ \mathbf{h}=\mathbf{0}(\bmod v)}} \frac{1}{r(\mathbf{h}, q)}<\frac{1}{v}\left(\frac{2}{\pi} \log q+\frac{7}{5}\right)^{d}
$$

for any divisor $v$ of $q$ with $1 \leq v<q$, and

$$
\sum_{h_{0} \in(-T / 2, T / 2]} \frac{1}{r\left(h_{0}, T\right)} \leq \frac{2}{\pi} \log T+\frac{7}{5} .
$$

\section{DISCREPANCY BOUNDS}

\subsection{Classical Discrepancy}

The following quantities play a crucial role in the analysis of the average discrepancy. For $1 \leq i \leq r$ and $\mathbf{z}_{n}^{(i)}, \mathbf{h} \in \mathbb{Z}^{d}$ with $\mathbf{h} \not \mathbf{0}\left(\bmod p_{i}\right)$, define

$$
B_{i}(\mathbf{h})=\max _{h^{\prime} \in \mathbb{Z}_{p_{i}}} \#\left\{0 \leq n<p_{i} \mid \mathbf{h} \cdot \mathbf{z}_{n}^{(i)} \equiv h^{\prime}\left(\bmod p_{i}\right)\right\} .
$$

Lemma 4 . Let $1 \leq T \leq m=p_{1} \cdots p_{r}, \mathbf{h} \in C_{d}(m)$, and $J=\{1 \leq i \leq r \mid \mathbf{h} \equiv$ $\left.\mathbf{0}\left(\bmod p_{i}\right)\right\}$. Then

$$
\sum_{\left(c_{1}, \ldots, c_{r}\right) \in \mathbb{Z}_{p_{1}}^{*} \times \cdots \times \mathbb{Z}_{p_{r}}^{*}}\left|\sum_{n=0}^{T-1} e\left(\sum_{i=1}^{r} \frac{c_{i}\left(\mathbf{h} \cdot \mathbf{z}_{n}^{(i)}\right)}{p_{i}}+\frac{n h_{0}}{T}\right)\right|^{2} \leq T m \prod_{i \in J} p_{i} \prod_{\substack{i=1 \\ i \neq J}}^{r} B_{i}(\mathbf{h}) .
$$


Proof. Repeating the proof of Eichenauer-Herrmann et al. [1997, Lemma 4], we obtain that the left side of (9) is at most

$$
\begin{aligned}
& \sum_{k, n=0}^{T-1}\left|\prod_{i=1}^{r} \sum_{c \in \mathbb{Z}_{p_{i}}} e\left(\frac{c\left(\mathbf{h} \cdot \mathbf{z}_{n}^{(i)}-\mathbf{h} \cdot \mathbf{z}_{k}^{(i)}\right)}{p_{i}}+\frac{h_{0}(n-k)}{T}\right)\right| \\
& =\sum_{k, n=0}^{T-1} \prod_{i=1}^{r}\left|\sum_{c \in \mathbb{Z}_{p_{i}}} e\left(\frac{c\left(\mathbf{h} \cdot \mathbf{z}_{n}^{(i)}-\mathbf{h} \cdot \mathbf{z}_{k}^{(i)}\right)}{p_{i}}\right)\right| \\
& =m \sum_{k=0}^{T-1} \#\left\{0 \leq n<T \mid \mathbf{h} \cdot \mathbf{z}_{n}^{(i)} \equiv \mathbf{h} \cdot \mathbf{z}_{k}^{(i)}\left(\bmod p_{i}\right), 1 \leq i \leq r\right\} .
\end{aligned}
$$

Next, applying the final calculations of the proof of Eichenauer-Herrmann et al. [1997, Lemma 4], we get the desired result.

In the following, we use the abbreviation (see (2), (3), and (7))

$$
\tilde{D}_{T ; c_{1}, \ldots, c_{r}}^{(d)}=\tilde{D}\left(\left(\mathbf{x}_{n}\right)_{n=0}^{T-1}\right) .
$$

Lemma 5. Let $1 \leq T \leq m$ and $m_{J}=\prod_{i \in J} p_{i}$ for subsets $J$ of $\{1, \ldots, r\}$. Then

$$
\begin{aligned}
& \frac{1}{\prod_{i=1}^{r}\left(p_{i}-1\right)} \sum_{\left(c_{1}, \ldots, c_{r}\right) \in \mathbb{Z}_{p_{i}}^{*} \times \ldots \times \mathbb{Z}_{p_{r}}^{*}} \tilde{D}_{T ; c_{1}, \ldots, c_{r}}^{(d)}
\end{aligned}
$$

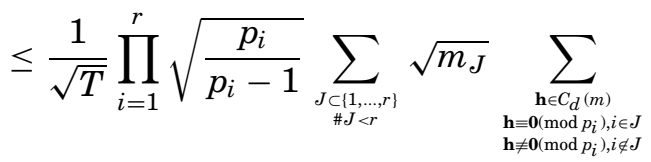

$$
\begin{aligned}
& \times \sum_{h_{0} \in(-T / 2, T / 2]} \frac{1}{r(\mathbf{h}, m) r\left(h_{0}, T\right)} \prod_{\substack{i=1 \\
i \notin J}}^{r} \sqrt{B_{i}(\mathbf{h})} .
\end{aligned}
$$

Proof. Define the left side of (11) by $\sigma$. Applying the Cauchy-Schwarz inequality, we obtain from (2), (3), (7) and (9) that

$$
\begin{aligned}
& \sigma \leq \frac{1}{T} \sum_{\mathbf{h} \in C_{d}(m)} \sum_{h_{0} \in(-T / 2, T / 2]} \frac{1}{r(\mathbf{h}, m) r\left(h_{0}, T\right)} \\
& \times\left(\frac{1}{\prod_{i=1}^{r}\left(p_{i}-1\right)} \sum_{\left(c_{1}, \ldots, c_{r}\right) \in \mathbb{Z}_{p_{1}}^{*} \times \cdots \times \mathbb{Z}_{p r}^{*}}\left|\sum_{n=0}^{T-1} e\left(\sum_{i=1}^{r} \frac{c_{i}\left(\mathbf{h} \cdot \mathbf{z}_{n}^{(i)}\right)}{p_{i}}+\frac{n h_{0}}{T}\right)\right|^{2}\right)^{1 / 2}
\end{aligned}
$$

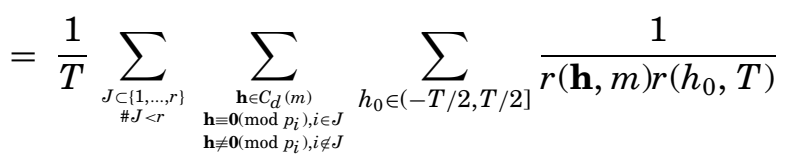

$$
\begin{aligned}
& \times\left(\frac{1}{\prod_{i=1}^{r}\left(p_{i}-1\right)} \sum_{\left(c_{1}, \ldots, c_{r}\right) \in \mathbb{Z}_{p_{i}}^{*} \times \cdots \times \mathbb{Z}_{p r}^{*}}\left|\sum_{n=0}^{T-1} e\left(\sum_{i=1}^{r} \frac{c_{i}\left(\mathbf{h} \cdot \mathbf{z}_{n}^{(i)}\right)}{p_{i}}+\frac{n h_{0}}{T}\right)\right|^{2}\right)^{1 / 2} .
\end{aligned}
$$

ACM Transactions on Modeling and Computer Simulation, Vol. 11, No. 3, July 2001. 
By Lemma 4, we have

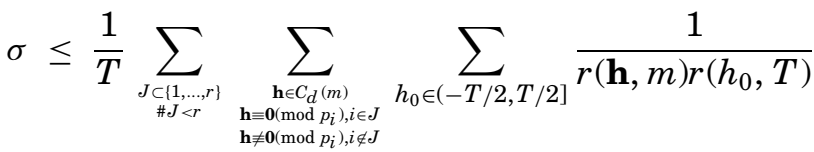

$$
\begin{aligned}
& \times\left(T \prod_{i=1}^{r} \frac{p_{i}}{p_{i}-1} m_{J} \prod_{\substack{i=1 \\
i \notin J}}^{r} B_{i}(\mathbf{h})\right)^{1 / 2} .
\end{aligned}
$$

Lemma 6. Let $1 \leq T \leq m$, and $B_{i}=\max \left\{B_{i}(\mathbf{h}) \mid \mathbf{h} \in \mathbb{Z}^{d}, \mathbf{h} \neq \equiv \mathbf{0}\left(\bmod p_{i}\right)\right\}$ for $1 \leq i \leq r$. Then

$$
\begin{aligned}
& \frac{1}{\prod_{i=1}^{r}\left(p_{i}-1\right)} \sum_{\left(c_{1}, \ldots, c_{r}\right) \in \mathbb{Z}_{p_{1}}^{*} \times \cdots \times \mathbb{Z}_{p_{r}}^{*}} \tilde{D}_{T ; c_{1}, \ldots, c_{r}}^{(d)} \\
& \quad \leq \frac{1}{\sqrt{T}} \prod_{i=1}^{r} \frac{\sqrt{p_{i} B_{i}}+1}{\sqrt{p_{i}-1}}\left(\frac{2}{\pi} \log m+\frac{7}{5}\right)^{d}\left(\frac{2}{\pi} \log T+\frac{7}{5}\right) .
\end{aligned}
$$

Proof. Using Lemma 5 and the two inequalities of Lemma 3 (with $q=m$ and $v=m_{J}$ ), we get that the left side of (12) is less than

$$
\frac{1}{\sqrt{T}} \prod_{i=1}^{r} \sqrt{\frac{p_{i}}{p_{i}-1}}\left(\frac{2}{\pi} \log T+\frac{7}{5}\right) \sum_{\substack{J \subset\{1, \ldots, r\} \\ \nexists J<r}} \frac{1}{\sqrt{m_{J}}}\left(\frac{2}{\pi} \log m+\frac{7}{5}\right)^{d} \prod_{\substack{i=1 \\ i \notin J}}^{r} \sqrt{B_{i}} .
$$

According to Eichenauer-Herrmann et al. [1997, p. 211],

$$
\prod_{i=1}^{r} \sqrt{\frac{p_{i}}{p_{i}-1}} \sum_{\substack{J \in\{1, \ldots r r\} \\ \sharp J<r}} \frac{1}{\sqrt{m_{J}}} \prod_{\substack{i=1 \\ i \notin J}}^{r} \sqrt{B_{i}} \leq \prod_{i=1}^{r} \frac{\sqrt{p_{i} B_{i}}+1}{\sqrt{p_{i}-1}} .
$$

Now, from (13), we obtain the desired result.

Basic Lemma Let $T_{j}=\left[m / 2^{j}\right], j=0,1,2, \ldots$ Then

$$
\begin{aligned}
& \frac{1}{\prod_{i=1}^{r}\left(p_{i}-1\right)} \sum_{\left(c_{1}, \ldots, c_{r}\right) \in \mathbb{Z}_{p_{1}}^{*} \times \cdots \times \mathbb{Z}_{p_{r}}^{*}} \sum_{0 \leq j \leq\left[\log _{2} m\right]-1} \tilde{D}_{T_{j} ; c_{1}, \ldots, c_{r}}^{(d)} \\
& \quad \times \frac{\sqrt{T_{j}}}{\left((2 / \pi) \log T_{j}+(7 / 5)\right)\left(\log _{2} T_{j}+3\right) \log ^{3 / 2}\left(\log _{2} T_{j}+3\right)} \\
& \leq 2 \prod_{i=1}^{r} \frac{\sqrt{p_{i} B_{i}}+1}{\sqrt{p_{i}-1}}\left(\frac{2}{\pi} \log m+\frac{7}{5}\right)^{d} .
\end{aligned}
$$

Proof. It is easy to see that $\log _{2} T_{j} \geq\left[\log _{2} m\right]-j, j \in\left[0,\left[\log _{2} m\right]\right]$. Hence

$$
\begin{aligned}
& \sum_{0 \leq j \leq\left[\log _{2} m\right]-1} \frac{1}{\left(\log _{2} T_{j}+3\right) \log ^{3 / 2}\left(\log _{2} T_{j}+3\right)} \leq \sum_{j=1}^{\infty} \frac{1}{(j+3) \log ^{3 / 2}(j+3)} \\
\leq & \int_{3}^{\infty} \frac{d x}{x(\log x)^{3 / 2}}=-\left.2(\log x)^{-1 / 2}\right|_{3} ^{\infty}<2 .
\end{aligned}
$$


By Lemma 6, we get the desired result.

Theorem 1. Let $0<\varepsilon \leq 1$. Then there exist more than $(1-\varepsilon) \prod_{i=1}^{r}\left(p_{i}-1\right)$ values of $\left(c_{1}, \ldots, c_{r}\right) \in \mathbb{Z}_{p_{1}}^{*} \times \cdots \times \mathbb{Z}_{p_{r}}^{*}$ such that for $N=1,2, \ldots, m$

$$
\begin{aligned}
& D_{N ; c_{1}, \ldots, c_{r}}^{(d)} \leq \frac{d}{m}+\frac{2 \sqrt{2}}{\varepsilon} \prod_{i=1}^{r} \frac{\sqrt{p_{i} B_{i}}+1}{\sqrt{p_{i}-1}} N^{-1 / 2}\left(\frac{2}{\pi} \log m+\frac{7}{5}\right)^{d} \\
& \times\left(\frac{2}{\pi} \log 2 N+\frac{7}{5}\right)\left(\log _{2} N+4\right) \log ^{3 / 2}\left(\log _{2} N+4\right) .
\end{aligned}
$$

Proof. Let,

$$
\begin{aligned}
\Omega_{\varepsilon}= & \left\{\left(c_{1}, \ldots, c_{r}\right) \in \mathbb{Z}_{p_{1}}^{*} \times \cdots \times \mathbb{Z}_{p_{r}}^{*} \mid \sum_{j=0}^{\left[\log _{2} m\right]-1}\right. \\
& \times \tilde{D}_{T_{j} ; c_{1}, \ldots, c_{r}}^{(d)} \frac{\sqrt{T_{j}}}{\left((2 / \pi) \log T_{j}+(7 / 5)\right)\left(\log _{2} T_{j}+3\right)} \\
& \left.\times \log ^{-3 / 2}\left(\log _{2} T_{j}+3\right) \leq \frac{2}{\varepsilon}\left(\frac{2}{\pi} \log m+\frac{7}{5}\right)^{d} \prod_{i=1}^{r} \frac{\sqrt{p_{i} B_{i}+1}}{\sqrt{p_{i}-1}}\right\} .
\end{aligned}
$$

It follows from the Basic Lemma that

$$
\# \Omega_{\varepsilon} \geq(1-\varepsilon) \prod_{i=1}^{r}\left(p_{i}-1\right) .
$$

For $N=1$, the statement (16) is evident.

Now let $N \in\left(T_{j_{0}+1}, T_{j_{0}}\right]$ for any $j_{0} \in\left[0,\left[\log _{2} m\right]-1\right]$, where $T_{j_{0}}=\left[m / 2^{j_{0}}\right]$.

By (4), (6), and (10), we have that for $\left(c_{1}, \ldots, c_{r}\right) \in \Omega_{\varepsilon}$

$$
\begin{aligned}
& D_{N ; c_{1}, \ldots, c_{r}}^{(d)} \leq \frac{d}{m}+\frac{T_{j_{0}}}{N} \tilde{D}_{T_{j_{0}} ; c_{1}, \ldots, c_{r}}^{(d)} \leq \frac{d}{m}+\frac{2 \sqrt{T_{j_{0}}}}{\varepsilon N} \prod_{i=1}^{r} \frac{\sqrt{p_{i} B_{i}}+1}{\sqrt{p_{i}-1}} \\
& \quad \times\left(\frac{2}{\pi} \log m+\frac{7}{5}\right)^{d}\left(\frac{2}{\pi} \log T_{j_{0}}+\frac{7}{5}\right)\left(\log _{2} T_{j_{0}}+3\right) \log ^{3 / 2}\left(\log _{2} T_{j_{0}}+3\right) .
\end{aligned}
$$

Bearing in mind that $2 N \geq 2\left(\left[m / 2^{j_{0}+1}\right]+1\right) \geq m / 2^{j_{0}} \geq T_{j_{0}}$, we obtain from (17) the assertion of Theorem 1.

The right side of (16) depends on the period $m$. We can obtain an estimate independently of the period $m$ by applying the Erdős-Turan-Koksma inequality (see Drmota and Tichy [1997, p. 15]):

$$
D\left(\left(\mathbf{x}_{n}\right)_{n=0}^{N-1}\right) \leq\left(\frac{3}{2}\right)^{d}\left(\frac{2}{H+1}+\sum_{\mathbf{0}<\|\mathbf{h}\| \leq H} \frac{1}{r(\mathbf{h})}\left|\frac{1}{N} \sum_{n=0}^{N-1} e\left(\mathbf{h} \cdot \mathbf{x}_{n}\right)\right|\right),
$$

where $r(\mathbf{h})=\prod_{i=1}^{d} \max \left(1,\left|h_{i}\right|\right)$ for $\mathbf{h}=\left(h_{1}, \ldots, h_{d}\right) \in Z^{d},\|\mathbf{h}\|=\max _{1 \leq i \leq d}\left|h_{i}\right|$, $H \geq 1$. For the smaller constant in (18), see Cochrane [1988].

Úsing (18) with $H=\left[\sqrt{T}_{j}\right]\left(j=0,1, \ldots,\left[\log _{2} m\right]-1\right)$ instead of Lemma 1 and repeating the above calculation, we get 
Theorem $1^{\prime}$. Let $0<\varepsilon \leq 1$. Then there exist more than $(1-\varepsilon) \prod_{i=1}^{r}\left(p_{i}-1\right)$ values of $\left(c_{1}, \ldots, c_{r}\right) \in \mathbb{Z}_{p_{1}}^{*} \times \cdots \times \mathbb{Z}_{p_{r}}^{*}$ such that

$$
\begin{aligned}
D_{N ; c_{1}, \ldots, c_{r}}^{(d)} & <\left(\frac{3}{2}\right)^{d} \frac{3}{\varepsilon} \prod_{i=1}^{r} \frac{\sqrt{p_{i} B_{i}}+1}{\sqrt{p_{i}-1}} N^{-1 / 2}(2 \log N+4)^{d+2} \log ^{3 / 2}\left(\log _{2} N+4\right) \\
\quad \text { for } \quad N & =1,2, \ldots, m
\end{aligned}
$$

It is easy to see that the estimate (19) is preferable to (16) for small parts of the period.

Remark 1. As in Levin [1989], we can obtain a discrepancy estimate for a large set of subsequences of a given pseudorandom sequence (see also Knuth [1981, pp. 154-160] on subsequences of a random sequence). Repeating the proof of Theorem 1, we get the following result for the arithmetic progression subsequences:

Let $a_{v} \geq 1, b_{v} \geq 0(v=1, \ldots, L)$ be integers, $a_{1} \cdots a_{L} \not \equiv 0\left(\bmod p_{i}\right)(i=$ $1, \ldots, r)$ and $0<\varepsilon \leq 1$. Then there exist more than $(1-\varepsilon) \prod_{i=1}^{r}\left(p_{i}-1\right)$ values of $\left(c_{1}, \ldots, c_{r}\right) \in \mathbb{Z}_{p_{1}}^{*} \times \cdots \times \mathbb{Z}_{p_{r}}^{*}$ such that

$$
\begin{aligned}
& D\left(\left(\mathbf{x}_{n a_{v}+b_{v}}\right)_{n=0}^{N-1}\right)<\left(\frac{3}{2}\right)^{d} \frac{3 L}{\varepsilon} \prod_{i=1}^{r} \frac{\sqrt{p_{i} B_{v i}}+1}{\sqrt{p_{i}-1}} N^{-1 / 2} \\
& \quad \times(2 \log N+4)^{d+2} \log ^{3 / 2}\left(\log _{2} N+4\right), \quad \text { with } \quad v=1, \ldots, L, \quad N=1, \ldots, m,
\end{aligned}
$$

where

$$
B_{v i}=\max _{\mathbf{h} \in \mathbb{Z}^{d}, \mathbf{h} \neq \mathbf{0}\left(\bmod p_{i}\right)} \max _{h^{\prime} \in \mathbb{Z}_{p_{i}}} \#\left\{0 \leq n<p_{i} \mid \mathbf{h} \cdot \mathbf{z}_{n a_{v}+b_{v}} \equiv h^{\prime}\left(\bmod p_{i}\right)\right\},
$$

\subsection{The Diaphony and the Choice of Parameters}

Let $\mathbf{u}_{0}, \ldots, \mathbf{u}_{N-1}$ be a finite sequence of points in $[0,1)^{d}$. The number

$$
F_{N}\left(\left(\mathbf{u}_{n}\right)_{n=0}^{N-1}\right)=\left(\frac{1}{N^{2}} \sum_{\mathbf{0} \neq \mathbf{h} \in \mathbf{Z}^{\mathbf{d}}} \frac{1}{r(\mathbf{h})^{2}}\left|\sum_{n=0}^{N-1} e\left(\mathbf{h} \cdot \mathbf{u}_{n}\right)\right|^{2}\right)^{1 / 2}
$$

is called the diaphony of the given sequence [Zinterhof 1976]. It is known that

$$
F_{N}\left(\left(\mathbf{u}_{n}\right)_{n=0}^{N-1}\right)=\left(\frac{1}{N^{2}} \sum_{n=0}^{N-1} \sum_{m=0}^{N-1}\left(\prod_{i=1}^{d} g\left(u_{n, i}-u_{m, i}\right)-1\right)\right)^{1 / 2},
$$

where $g(u)=1-\left(\pi^{2} / 6\right)+\left(\pi^{2} / 2\right)(1-2\{u\})^{2}$ (see Drmota and Tichy [1997, p. 24] and Zinterhof [1976]).

Diaphony is closely related to discrepancy (see Lev [1995] and Stegbuchner [1979]). The computational complexity of the discrepancy of the sequence $\left(\mathbf{u}_{n}\right)_{n=0}^{N-1}$ is $O\left(N^{d}\right)$. In the case of diaphony, it is $O\left(d N^{2}\right)$. This renders it an interesting alternative to the discrepancy in higher dimensions. 
Let $F_{N ; c_{1}, \ldots, c_{r}}^{(d)}$ be the diaphony of the sequence $\left(\mathbf{x}_{n}\right)_{n=0}^{N-1}$ in (2)-(3) and

$$
\tilde{F}_{T ; c_{1}, \ldots, c_{r}}^{(d)}=\left(\frac{1}{T^{2}} \sum_{h_{0} \in(-T / 2, T / 2]} \sum_{\mathbf{0} \neq \mathbf{h} \in \mathbf{Z}^{\mathbf{d}}} \frac{1}{r(\mathbf{h})^{2} r\left(h_{0}, T\right)}\left|\sum_{n=0}^{T-1} e\left(\mathbf{h} \cdot \mathbf{x}_{n}+\frac{h_{0} n}{T}\right)\right|^{2}\right)^{1 / 2} .
$$

We have from (20) and (21) that

$$
\begin{aligned}
\tilde{F}_{T ; c_{1}, \ldots, c_{r}}^{(d)}= & \left(\frac{1}{T^{2}} \sum_{h_{0} \in(-T / 2, T / 2]} \frac{1}{r\left(h_{0}, T\right)} \sum_{n=0}^{T-1} \sum_{m=0}^{T-1}\left(\prod_{i=1}^{d} g\left(x_{n, i}-x_{m, i}\right)-1\right)\right. \\
& \left.\times e\left(\frac{h_{0}(n-m)}{T}\right)\right)^{1 / 2} .
\end{aligned}
$$

Using Lemma 2 and the Cauchy-Schwarz inequality, we obtain, for $1 \leq N \leq T$,

$$
\begin{aligned}
& \left|\sum_{n=0}^{N-1} e\left(\mathbf{h} \cdot \mathbf{x}_{n}\right)\right|^{2} \\
& \quad \leq\left(\sum_{h_{0} \in(-T / 2, T / 2]} \frac{1}{r\left(h_{0}, T\right)}\left|\sum_{n=0}^{T-1} e\left(\mathbf{h} \cdot \mathbf{x}_{n}+\frac{h_{0} n}{T}\right)\right|\right)^{2} \\
& \quad \leq \sum_{h_{0} \in(-T / 2, T / 2]} \frac{1}{r\left(h_{0}, T\right)}\left(\sum_{h_{0} \in(-T / 2, T / 2]} \frac{1}{r\left(h_{0}, T\right)}\left|\sum_{n=0}^{T-1} e\left(\mathbf{h} \cdot \mathbf{x}_{n}+\frac{h_{0} n}{T}\right)\right|^{2}\right) .
\end{aligned}
$$

Hence, from (20), (22) and Lemma 3, we get

$$
\left(N F_{N ; c_{1}, \ldots, c_{r}}^{(d)}\right)^{2} \leq\left(\frac{2}{\pi} \log T+\frac{7}{5}\right)\left(T \tilde{F}_{T ; c_{1}, \ldots, c_{r}}^{(d)}\right)^{2} \quad \text { for } \quad 1 \leq N \leq T .
$$

Lemma 7. With the notation defined above, we have for $1 \leq T \leq m$

$$
\begin{aligned}
& \frac{1}{\prod_{i=1}^{r}\left(p_{i}-1\right)} \sum_{\left(c_{1}, \ldots, c_{r}\right) \in \mathbb{Z}_{p_{1}^{*}}^{*} \times \cdots \times \mathbb{Z}_{p_{r}}^{*}} \tilde{F}_{T ; c_{1}, \ldots, c_{r}}^{(d)} \\
& \leq\left(\frac{1}{T}\left(\frac{2}{\pi} \log T+\frac{7}{5}\right) 12 d\left(1+\frac{\pi^{2}}{3}\right)^{d-1} \prod_{i=1}^{r} \frac{\left(p_{i}+1\right) B_{i}}{p_{i}-1}\right)^{1 / 2} .
\end{aligned}
$$

Proof. We define the left side of (25) by $\sigma$. Applying the Cauchy-Schwarz inequality, we get from (22), (2), and (3) that

$$
\begin{aligned}
\sigma^{2} \leq & \frac{1}{T^{2}} \sum_{h_{0} \in(-T / 2, T / 2]} \sum_{\mathbf{0} \neq \mathbf{h} \in \mathbf{Z}^{\mathbf{d}}} \frac{1}{r(\mathbf{h})^{2} r\left(h_{0}, T\right)} \\
& \times \frac{1}{\prod_{i=1}^{r}\left(p_{i}-1\right)} \sum_{\left(c_{1}, \ldots, c_{r}\right) \in \mathbb{Z}_{p_{1}}^{*} \times \cdots \times \mathbb{Z}_{p_{r}}^{*}}\left|\sum_{n=0}^{T-1} e\left(\sum_{i=1}^{r} \frac{c_{i}\left(\mathbf{h} \cdot \mathbf{z}_{n}^{(i)}\right)}{p_{i}}+\frac{h_{0} n}{T}\right)\right|^{2} .
\end{aligned}
$$

It is easy to see that

$$
\sigma^{2} \leq \sigma_{1}+\sigma_{2}
$$

ACM Transactions on Modeling and Computer Simulation, Vol. 11, No. 3, July 2001. 
where

$$
\begin{aligned}
\sigma_{1}= & \frac{1}{T^{2}} \sum_{h_{0} \in(-T / 2, T / 2]} \sum_{\mathbf{h} \in C_{d}(m)} \frac{1}{r(\mathbf{h})^{2} r\left(h_{0}, T\right)} \frac{1}{\prod_{i=1}^{r}\left(p_{i}-1\right)} \sum_{\left(c_{1}, \ldots, c_{r}\right) \in \mathbb{Z}_{p_{1}}^{*} \times \cdots \times \mathbb{Z}_{p r}^{*}} \\
& \times\left|\sum_{n=0}^{T-1} e\left(\sum_{i=1}^{r} \frac{c_{i}\left(\mathbf{h} \cdot \mathbf{z}_{n}^{(i)}\right)}{p_{i}}+\frac{h_{0} n}{T}\right)\right|^{2},
\end{aligned}
$$

and

$$
\sigma_{2}=\sum_{h_{0} \in(-T / 2, T / 2]} \sum_{\mathbf{h} \in Z^{d} \backslash\left(\mathbf{0} \cup C_{d}(m)\right)} \frac{1}{r(\mathbf{h})^{2} r\left(h_{0}, T\right)} .
$$

According to Hellekalek and Niederreiter [1998, pp. 49-51],

$$
\sum_{\mathbf{0} \neq \mathbf{h} \in \mathbf{Z}^{\mathbf{d}}, \mathbf{h}=\mathbf{0} \bmod \mathbf{M}} \frac{1}{r(\mathbf{h})^{2}}=\left(1+\frac{\pi^{2}}{3 M^{2}}\right)^{d}-1, \quad M=1,2, \ldots,
$$

and

$$
\sum_{\mathbf{h} \in Z^{d}} \frac{1}{r(\mathbf{h})^{2}}-\sum_{\mathbf{h} \in \mathbf{0} \cup C_{d}(M)} \frac{1}{r(\mathbf{h})^{2}}<\frac{12\left(\left(1+\pi^{2} / 3\right)^{d}-1\right)}{\pi^{2}(M-1)}, \quad M=2,3, \ldots
$$

By the mean-value theorem of calculus, we obtain bounds

$$
\sum_{\mathbf{0} \neq \mathbf{h} \in \mathbf{Z}^{\mathbf{d}}, \mathbf{h}=\mathbf{0} \bmod \mathbf{M}} \frac{1}{r(\mathbf{h})^{2}} \leq d \frac{\pi^{2}}{3 M^{2}}\left(1+\frac{\pi^{2}}{3}\right)^{d-1}, \quad M=1,2, \ldots,
$$

and

$$
\sum_{\mathbf{h} \in Z^{d}} \frac{1}{r(\mathbf{h})^{2}}-\sum_{\mathbf{h} \in \mathbf{0} \cup C_{d}(M)} \frac{1}{r(\mathbf{h})^{2}}<\frac{4 d\left(1+\pi^{2} / 3\right)^{d-1}}{M-1}, \quad M=2,3, \ldots
$$

It follows from (28) and Lemma 3 that

$$
\sigma_{2} \leq \frac{8 d\left(1+\pi^{2} / 3\right)^{d-1}}{m}\left(\frac{2}{\pi} \log T+\frac{7}{5}\right) .
$$

Let $J=\left\{1 \leq i \leq r \mid \mathbf{h} \equiv \mathbf{0}\left(\bmod p_{i}\right)\right\}$, and $m_{J}=\prod_{i \in J} p_{i}$.

Applying Lemma 4, we get from (27) that

$$
\begin{aligned}
\sigma_{1} & \leq \frac{1}{T^{2}} \sum_{h_{0} \in(-T / 2, T / 2]} \sum_{\mathbf{h} \in C_{d}(m)} \frac{1}{r(\mathbf{h})^{2} r\left(h_{0}, T\right)} T \prod_{i=1}^{r} \frac{p_{i}}{p_{i}-1} \prod_{i \in J} p_{i} \prod_{\substack{i=1 \\
i \notin J}}^{r} B_{i}(\mathbf{h}) \\
& =\frac{1}{T} \sum_{h_{0} \in(-T / 2, T / 2]} \prod_{i=1}^{r} \frac{p_{i}}{p_{i}-1} \sum_{\substack{J \subset 1, \ldots, r) \\
\text { HJJ }}} m_{J} \sum_{\substack{\mathbf{h} \in C_{d}(m) \\
\text { h=od }(m), i \in J \\
\mathbf{h} \neq 0\left(\bmod p_{i}\right), i \notin J}} \frac{1}{r(\mathbf{h})^{2} r\left(h_{0}, T\right)} \prod_{\substack{i=1 \\
i \notin J}}^{r} B_{i}(\mathbf{h}) .
\end{aligned}
$$

Using Lemma 3 and (29) with $M=m_{j}$, we have that

$$
\sigma_{1} \leq \frac{1}{T}\left(\frac{2}{\pi} \log T+\frac{7}{5}\right) \prod_{i=1}^{r} \frac{p_{i}}{p_{i}-1} \sum_{\substack{J \subset 1, \ldots, r\} \\ \sharp J \backslash r}} \frac{d \pi^{2}}{3 m_{J}}\left(1+\frac{\pi^{2}}{3}\right)^{d-1} \prod_{i=1}^{r} B_{i},
$$


where $B_{i}=\max \left\{B_{i}(\mathbf{h}) \mid \mathbf{h} \in \mathbb{Z}^{d}, \mathbf{h} \not \equiv \mathbf{0}\left(\bmod p_{i}\right)\right\}$ for $1 \leq i \leq r$.

Bearing in mind that

$$
\sum_{J \subset\{1, \ldots, r\}, \# J<r} \frac{1}{m_{J}}<\prod_{i=1}^{r}\left(1+\frac{1}{p_{i}}\right),
$$

we get

$$
\sigma_{1} \leq \frac{1}{T}\left(\frac{2}{\pi} \log T+\frac{7}{5}\right) 4 d\left(1+\frac{\pi^{2}}{3}\right)^{d-1} \prod_{i=1}^{r} \frac{\left(p_{i}+1\right) B_{i}}{p_{i}-1}
$$

Now, by (26) and (30), we obtain that

$$
\sigma^{2} \leq \frac{1}{T}\left(\frac{2}{\pi} \log T+\frac{7}{5}\right) 12 d\left(1+\frac{\pi^{2}}{3}\right)^{d-1} \prod_{i=1}^{r} \frac{\left(p_{i}+1\right) B_{i}}{p_{i}-1} .
$$

This is the desired result.

We obtain from (15)

Corollary 2. Let $T_{j}=\left[m / 2^{j}\right], j=0,1,2, \ldots$ Then

$$
\begin{aligned}
& \frac{1}{\prod_{i=1}^{r}\left(p_{i}-1\right)} \sum_{\left(c_{1}, \ldots, c_{r}\right) \in \mathbb{Z}_{p_{1}}^{*} \times \cdots \times \mathbb{Z}_{p_{r}}^{*}} \sum_{\mathbf{0} \leq j \leq\left[\log _{2} m\right]-1} \tilde{F}_{T_{j} ; c_{1}, \ldots, c_{r}}^{(d)} \\
& \quad \times \frac{\sqrt{T_{j}}}{\left((2 / \pi) \log T_{j}+(7 / 5)\right)^{1 / 2}\left(\log _{2} T_{j}+3\right) \log ^{3 / 2}\left(\log _{2} T_{j}+3\right)} \\
& \quad \leq 2\left(12 d\left(1+\frac{\pi^{2}}{3}\right)^{d-1} \prod_{i=1}^{r} \frac{\left(p_{i}+1\right) B_{i}}{p_{i}-1}\right)^{1 / 2} .
\end{aligned}
$$

Theorem 2. Let $0<\varepsilon \leq 1$. Then there exist more than $(1-\varepsilon) \prod_{i=1}^{r}\left(p_{i}-1\right)$ values of $\left(c_{1}, \ldots, c_{r}\right) \in \mathbb{Z}_{p_{1}}^{*} \times \cdots \times \mathbb{Z}_{p_{r}}^{*}$ such that for $N=1,2, \ldots, m$

$$
\begin{aligned}
& F_{N ; c_{1}, \ldots, c_{r}}^{(d)} \leq \frac{4}{\epsilon}\left(6 d\left(1+\frac{\pi^{2}}{3}\right)^{d-1} \prod_{i=1}^{r} \frac{\left(p_{i}+1\right) B_{i}}{p_{i}-1}\right)^{1 / 2} \\
& \quad \times N^{-1 / 2}\left(\frac{2}{\pi} \log 2 N+\frac{7}{5}\right)\left(\log _{2} N+4\right) \log ^{3 / 2}\left(\log _{2} N+4\right)
\end{aligned}
$$

Proof. Let

$$
\begin{aligned}
\Omega_{\varepsilon}= & \left\{\left(c_{1}, \ldots, c_{r}\right) \in \mathbb{Z}_{p_{1}}^{*} \times \cdots \times\left.\mathbb{Z}_{p_{r}}^{*}\right|_{0 \leq j \leq\left[\log _{2} m\right]-1}\right. \\
& \times \tilde{F}_{T_{j} ; c_{1}, \ldots, c_{r}}^{(d)} \frac{\sqrt{T_{j}}}{\left((2 / \pi) \log T_{j}+(7 / 5)\right)^{1 / 2}}\left(\log _{2} T_{j}+3\right)^{-1} \log ^{-3 / 2}\left(\log _{2} T_{j}+3\right) \\
\leq & \left.\frac{2}{\epsilon}\left(12 d\left(1+\frac{\pi^{2}}{3}\right)^{d-1} \prod_{i=1}^{r} \frac{\left(p_{i}+1\right) B_{i}}{p_{i}-1}\right)^{1 / 2}\right\} .
\end{aligned}
$$

ACM Transactions on Modeling and Computer Simulation, Vol. 11, No. 3, July 2001. 
It follows from Corollary 2 that

$$
\# \Omega_{\varepsilon} \geq(1-\varepsilon) \prod_{i=1}^{r}\left(p_{i}-1\right) .
$$

For $N=1$, the statement (31) is evident.

Let $N \in\left(T_{j_{0}+1}, T_{j_{0}}\right]$ for any $j_{0} \in\left[0,\left[\log _{2} m\right]-1\right]$, where $T_{j_{0}}=\left[m / 2^{j_{0}}\right]$.

By (24), we have that for $\left(c_{1}, \ldots, c_{r}\right) \in \Omega_{\varepsilon}$

$$
\begin{aligned}
F_{N ; c_{1}, \ldots, c_{r}}^{(d)} \leq & \left(\frac{2}{\pi} \log T_{j_{0}}+\frac{7}{5}\right)^{1 / 2} \frac{T_{j_{0}}}{N} \tilde{F}_{T_{j_{0}} ; c_{1}, \ldots, c_{r}}^{(d)} \\
\leq & \frac{2}{\epsilon}\left(\frac{2}{\pi} \log T_{j_{0}}+\frac{7}{5}\right)\left(\log _{2} T_{j_{0}}+3\right) \log ^{3 / 2}\left(\log _{2} T_{j_{0}}+3\right) \frac{\sqrt{T_{j_{0}}}}{N} \\
& \times\left(12 d\left(1+\frac{\pi^{2}}{3}\right)^{d-1} \prod_{i=1}^{r} \frac{\left(p_{i}+1\right) B_{i}}{p_{i}-1}\right)^{1 / 2} .
\end{aligned}
$$

Taking into account that $2 N \geq T_{j_{0}}$, we obtain the assertion of Theorem 2 .

We choose the parameters $\left(c_{1}, \ldots, c_{r}\right) \in \mathbb{Z}_{p_{1}}^{*} \times \cdots \times \mathbb{Z}_{p_{r}}^{*}$, minimizing the expression

$$
\sum_{j=0}^{\left[\log _{2} m\right]} \sqrt{T_{j}}\left(\log _{2} T_{j}+3\right)^{-3} \tilde{F}_{T_{j} ; c_{1}, \ldots, c_{r}}^{(d)}, \quad \text { with } \quad T_{j}=\left[m / 2^{j}\right], j=0,1,2, \ldots
$$

According to (23), we need for this $O\left(d m^{4}(\log m)^{3}\right)$ arithmetic operations.

It should be noted that the period $m$ is usually more than $10^{9}$ and it is not realistic to perform $d m^{4}(\log m)^{3}$ arithmetic operations with conventional computers. To reduce the number of operations, we propose the following heuristic procedure:

Let $\mathbf{x}=\left(x_{1}, \ldots, x_{d}\right), J_{\mathbf{x}}=\left[0, x_{1}\right) \times \cdots \times\left[0, x_{d}\right)$, and

$$
D^{2}\left(\left(\mathbf{t}_{n}\right)_{n=0}^{N-1}\right)=\left(\int_{[0,1)^{d}}\left(F_{N}\left(J_{\mathbf{x}}\right)-x_{1} \cdots x_{d}\right)^{2} d \mathbf{x}\right)^{1 / 2}
$$

is the $L_{2}$-discrepancy of the sequence $\left(\mathbf{t}_{n}\right)_{n=0}^{N-1}$ in the $d$-dimensional unit cube $[0,1)^{d}$ (see the definition of the discrepancy in the introduction). According to Heinrich [1996] and Drmota and Tichy [1997, p. 372] there is an algorithm, which computes $L_{2}$-discrepancy of the sequence $\left(\mathbf{t}_{n}\right)_{n=0}^{N-1}$ with $O\left(N(\log N)^{d}\right)$ arithmetic operations.

Now let $D_{N ; c_{1}, \ldots, c_{r}}^{(1 ; d+1}\left(D_{N ; c_{1}, \ldots, c_{r}}^{(2 ; d+1)}\right)$ be the discrepancy $\left(L_{2}-\right.$ discrepancy) of the $(d+1)$-dimensional sequence $\left(\mathbf{x}_{n}, n / N\right)_{n=0}^{N-1}$, where $\left(\mathbf{x}_{n}\right)_{n \geq 0}(3)$ is the sequence of $d$-dimensional vectors of compound pseudorandom numbers.

Using the well-known relation between discrepancies of $d$-dimensional sequences $\left(\mathbf{t}_{n}\right)_{n=0}^{N-1}$ and $d+1$-dimensional sequences $\left(\mathbf{t}_{n}, n / T\right)_{n=0}^{T-1}$ (see, for example, Levin [1989, p. 101]), we obtain

$$
D_{N ; c_{1}, \ldots, c_{r}}^{(d)} \leq \frac{T D_{T ; c_{1}, \ldots, c_{r}}^{(1 ; d+1)}}{N} \quad \text { for } \quad 1 \leq N \leq T .
$$


We use this inequality instead of Corollary 1 to get a slightly different variant of the proof of Theorem $1^{\prime}$. To obtain the discrepancy estimate as in (19) (or as in (16)) it is enough to minimize $M_{\epsilon ; c_{1}, \ldots, c_{r}}^{(1 ; d+1)}$ over $\left(c_{1}, \ldots, c_{r}\right) \in \mathbb{Z}_{p_{1}}^{*} \times \cdots \times \mathbb{Z}_{p_{r}}^{*}$, with

$$
M_{\epsilon ; c_{1}, \ldots, c_{r}}^{(i, d+1)}=\sum_{0 \leq j \leq\left[\log _{2} m\right]-1} D_{T_{j} ; c_{1}, \ldots, c_{r}}^{(i, d+1)} \frac{\sqrt{T_{j}}}{\left(\log _{2} T_{j}+3\right)^{d+2}\left(\log \left(\log _{2} T_{j}+3\right)\right)^{3 / 2}},
$$

where $i=1,2, T_{j}=\left[m / 2^{j}\right]$ and $j=1,2, \ldots$ It is easy to see that $M_{\epsilon ; c_{1}, \ldots, c_{r}}^{(2 ; d+1)} \leq$ $M_{\epsilon ; c_{1}, \ldots, c_{r}}^{(1 ;+1)}$. Bearing in mind that the $L_{2}$-discrepancy $D_{N ; c_{1}, \ldots, c_{r}}^{(2 ; d+1)}$ is closely related to the discrepancy $D_{N ; c_{1}, \ldots, c_{r}}^{(1 ; d+1)}$, we get that if $\left(c_{1}, \ldots, c_{r}\right) \in \mathbb{Z}_{p_{1}}^{*} \times \cdots \times \mathbb{Z}_{p_{r}}^{*}$ minimizes $M_{\epsilon ; c_{1}, \ldots, c_{r}}^{(2 ; j+1)}$, then $M_{\epsilon ; c_{1}, \ldots, c_{r}}^{(1 ; d+1)}$ should not be too large relative to the minimum of $M_{\epsilon ; c_{1}, \ldots, c_{r}}^{(1 ; d+1)}$ over $\left(c_{1}, \ldots, c_{r}\right) \in \mathbb{Z}_{p_{1}}^{*} \times \cdots \times \mathbb{Z}_{p_{r}}^{*}$. Thus, we propose (heuristically) to minimize $M_{\epsilon ; c_{1}, \ldots, c_{r}}^{(2 ; d+1)}$ over $\left(c_{1}, \ldots, c_{r}\right) \in \mathbb{Z}_{p_{1}}^{*} \times \cdots \times \mathbb{Z}_{p_{r}}^{*}$ instead of minimizing the $M_{\epsilon ; c_{1}, \ldots, c_{r}}^{(1 ; d+\epsilon)}$. According to Heinrich [1996] and Drmota and Tichy [1997, p. 372], we need $O\left(m(\log m)^{d+2}\right)$ arithmetic operations to compute $M_{\epsilon ; c_{1}, \ldots, c_{r}}^{(2 ; d)}$, and $O\left(m^{2}(\log m)^{d+2}\right)$ arithmetic operations to perform the full minimization. This minimization evidently admits a parallelization. To reduce the number of operations further we propose using a random search procedure (see Niederreiter [1992, chap. 6]) instead of the full minimization. Alternatively, it is possible to use the partial minimization over $\left(b_{1}, \ldots, b_{r}\right) \in \mathbb{Z}_{q_{1}}^{*} \times \cdots \times \mathbb{Z}_{q_{r}}^{*}$, taking $c_{i}=\left[p_{i}\left\{b_{i} / q_{i}\right\}\right], i=1, \ldots, r$, where integers $q_{i}$ are small relatively $p_{i}, i=1, \ldots, r$. In this way, we need $O\left(q m(\log m)^{d+2}\right)$ arithmetic operations for choosing the parameters $c_{1}, \ldots, c_{r}$, where $q=q_{1} \cdots q_{r}$. We propose to use this procedure for not too large dimension $d$ and period $m$ (say $d \leq 5, m \leq 10^{9}$ ).

\section{APPLICATION}

\subsection{The Compound Nonlinear Congruential Method}

The nonlinear congruential method is introduced in Eichenauer et al. [1988]. The compound version of this method is proposed in Eichenauer-Herrmann [1994a]. The following $k$-dimensional variant of this method is investigated in Eichenauer-Herrmann and Niederreiter [1997]. For the average-case analysis, see Eichenauer-Herrmann and Larcher [1996] and Eichenauer-Herrmann and Niederreiter [1997]. For $i \in\{1, \ldots, r\}, j \in\{1, \ldots, k\}$, let $g_{i j}: \mathbb{Z} \rightarrow \mathbb{Z}_{p_{i}}$ be a monic permutation polynomial of $\mathbb{Z}_{p_{i}}$ with degree $d_{i j}$ as a polynomial over $\mathbb{Z}_{p_{i}}$ and with $g_{i j}(0)=0$, where $3 \leq d_{i j} \leq p_{i}-2$ is assumed in order to avoid uninteresting cases. Let $\left(y_{n j}^{(i)}\right)_{n \geq 0}$ with

$$
y_{n j}^{(i)} \equiv g_{i j}(n)+b_{i}\left(\bmod p_{i}\right), \quad n \geq 0
$$

be the corresponding (ordinary) nonlinear congruential sequence of elements of $\mathbb{Z}_{p_{i}}$ and let $\left(x_{n j}^{(i)}\right)_{n \geq 0}$ with

$$
x_{n j}^{(i)}=c_{i} z_{n j}^{(i)}, \quad z_{n j}^{(i)}=\frac{y_{n j}^{(i)}}{p_{i}}, \quad c_{i} \not \equiv 0\left(\bmod p_{i}\right), \quad n \geq 0
$$

be the corresponding stream of (ordinary) nonlinear congruential pseudorandom numbers. 
Now in the compound approach, the corresponding stream of $k$-dimensional nonlinear congruential pseudorandom vectors in $[0,1)^{k}$ is defined by $\mathbf{x}_{n}=$ $\left(x_{n 1}, \ldots, x_{n k}\right)$, with $x_{n j} \equiv x_{n j}^{(1)}+\cdots+x_{n j}^{(r)}(\bmod 1), n \geq 0$. Since the primes $p_{1}, \ldots, p_{r}$ are distinct and $\left(g_{i j}\right),(i=1, \ldots, r ; j=1, \ldots, k)$ are permutation polynomials, the sequence $\left(\mathbf{x}_{n}\right)_{n \geq 0}$ is purely periodic with period length $m=p_{1} \cdots p_{r}$.

In the following, mutual statistical independence properties of the stream $\left(\mathbf{x}_{n}\right)_{n \geq 0}$ of uniform pseudorandom vectors are studied based on the equidistribution behavior of the $s$-tuples

$$
\left(\mathbf{x}_{n}, \ldots, \mathbf{x}_{n+s-1}\right) \in[0,1)^{k s}, \quad n \geq 0,
$$

which can be analyzed by the discrepancy of the corresponding point sets in $[0,1)^{k s}$.

Theorem 3. For $i \in\{1, \ldots, r\}$ let the polynomials $\left(g_{i j}(s z+v-1)\right)(1 \leq j \leq$ $k, 1 \leq v \leq s)$ be linearly independent over $\mathbb{Z}_{p_{i}} ; \varepsilon \in(0,1], d_{i}=\max _{1 \leq j \leq k} d_{i j}$. Then there exist more than $(1-\varepsilon) \prod_{i=1}^{r}\left(p_{i}-1\right)$ values of $\left(c_{1}, \ldots, c_{r}\right) \in \mathbb{Z}_{p_{1}}^{*} \times \cdots \times \mathbb{Z}_{p_{r}}^{*}$ such that

$$
\begin{aligned}
& D_{N ; c_{1}, \ldots, c_{r}}^{(k s)}<\left(\frac{3}{2}\right)^{k s} \frac{3}{\varepsilon} \prod_{i=1}^{r}\left(\sqrt{d_{i}+1}+0.5\right) N^{-1 / 2}(2 \log N+4)^{k s+2} \\
& \quad \times \log ^{3 / 2}\left(\log _{2} N+4\right), \quad N=1,2, \ldots, m .
\end{aligned}
$$

Proof. For $i \in\{1, \ldots, r\}$ and $\mathbf{h} \in \mathbb{Z}^{k s}$, let

$$
Q^{(i)}(\mathbf{h}, z) \equiv \sum_{j=1}^{k} \sum_{v=1}^{s} h_{i j} g_{i j}(s z+v-1)\left(\bmod p_{i}\right) .
$$

We have from the condition of the theorem that for $\mathbf{h} \neq \mathbf{0}\left(\bmod p_{i}\right), 1 \leq$ $\operatorname{deg}\left(Q^{(i)}(\mathbf{h}, z)\right) \leq d_{i}$. Hence

$$
B_{i}=\max _{1 \leq h^{\prime} \leq p_{i}} \#\left\{0 \leq n<p_{i} \mid Q^{(i)}(\mathbf{h}, z) \equiv h^{\prime}\left(\bmod p_{i}\right)\right\} \leq d_{i} .
$$

Taking into account that $3 \leq d_{i} \leq p_{i}-2(i=1, \ldots, r)$, we have that

$$
\frac{\sqrt{p_{i} B_{i}}+1}{\sqrt{p_{i}-1}} \leq \sqrt{d_{i}+1}+0.5 \text {. }
$$

Now, applying Theorem $1^{\prime}$, we get the assertion of Theorem 3 .

\subsection{The Compound Inversive Method}

The inversive congruential method is introduced in Eichenauer and Lehn [1986]. The compound version of this method is analyzed in EichenauerHerrmann [1994b]. For the average-case analysis, see Eichenauer-Herrmann and Emmerich [1996]. Let $p_{1}, \ldots, p_{r} \geq 5$ be distinct primes. For $1 \leq i \leq r$, let $a_{i} \in \mathbb{Z}_{p_{i}}^{*}=\mathbb{Z}_{p_{i}} \backslash\{0\}$ and let $\left(z_{n}^{(i)}\right)_{n \geq 0}$ be a sequence in $\mathbb{Z}_{p_{i}}$ with

$$
z_{n+1}^{(i)} \equiv a_{i}\left(z_{n}^{(i)}\right)^{-1}+1\left(\bmod p_{i}\right), \quad n \geq 0,
$$


where $z^{-1}$ denotes the multiplicative inverse of $z$ in $\mathbb{Z}_{p_{i}}^{*}$ and $0^{-1}=0$. Obviously, the sequence $\left(z_{n}^{(i)}\right)_{n \geq 0}$ is always purely periodic and $p_{i}$ is the maximum possible period length. Let $\mathbb{M}_{p_{i}}$ be the set of all $a_{i} \in \mathbb{Z}_{p_{i}}^{*}$ which belong to sequences with period length $p_{i}$. The set $\mathbb{M}_{p_{i}}$ is always nonvoid and its elements can be characterized by properties of the polynomial $x^{2}-x-a_{i} \in \mathbb{Z}_{p_{i}}[x]$ (cf. Niederreiter [1992]). In the following, let $a_{i} \in \mathbb{M}_{p_{i}}, c_{i} \in \mathbb{Z}_{p_{i}}^{*}$ and

$$
x_{n}^{(i)}=\frac{c_{i} z_{n}^{(i)}}{p_{i}} \in[0,1), \quad n \geq 0,
$$

be the corresponding stream of (ordinary) inversive congruential pseudorandom numbers.

Now, a sequence $\left(x_{n}\right)_{n \geq 0}$ of compound inversive congruential pseudorandom numbers in the interval $[0,1)$ is defined by

$$
x_{n} \equiv x_{n}^{(1)}+\cdots+x_{n}^{(r)}(\bmod 1), \quad n \geq 0 .
$$

Since the primes $p_{1}, \ldots, p_{r}$ are distinct, the sequence $\left(x_{n}\right)_{n \geq 0}$ is purely periodic with period length $m=p_{1} \cdots p_{r}$ and $x_{0}, x_{1}, \ldots, x_{m-1}$ runs through all rationals in $[0,1)$ with denominator $m$.

In the following, nonoverlapping $s$-tuples of $k$-dimensional vectors $\left(\mathbf{x}_{s n}, \ldots\right.$, $\left.\mathbf{x}_{s n+s-1}\right)$ with $\mathbf{x}_{n}=\left(x_{k n}, x_{k n+1}, \ldots, x_{k n+k-1}\right) \in[0,1)^{k}, n \geq 0$, of compound inversive congruential pseudorandom numbers are considered.

Theorem 4. Let $1 \leq k s<\min \left(p_{1}, \ldots, p_{r}\right)$ and $\varepsilon \in(0,1]$. Then there are more than $(1-\varepsilon) \prod_{i=1}^{r}\left(p_{i}-1\right)$ values of $\left(c_{1}, \ldots, c_{r}\right) \in \mathbb{Z}_{p_{i}}^{*} \times \cdots \times \mathbb{Z}_{p_{r}}^{*}$ with

$$
\begin{aligned}
& D_{N ; c_{1}, \ldots, c_{r}}^{(k s)}<\left(\frac{3}{2}\right)^{k s} \frac{3}{\varepsilon}(\sqrt{2 k s+1}+0.5)^{r} N^{-1 / 2}(2 \log N+4)^{k s+2} \\
& \quad \times \log ^{3 / 2}\left(\log _{2} N+4\right), \quad N=1,2, \ldots, m .
\end{aligned}
$$

Proof. For $1 \leq i \leq r, 1 \leq s_{1}<p_{i}, h_{0} \in \mathbb{Z}_{p_{i}}$, and $\mathbf{h} \in \mathbb{Z}^{s_{1}}, \mathbf{h} \neq \equiv \mathbf{0}\left(\bmod p_{i}\right)$, it follows from Eichenauer-Herrmann and Emmerich [1996], Lemma 4 that

$$
\#\left\{0 \leq n<p_{i} \mid \mathbf{h} \mathbf{z}_{n}^{(i)} \equiv h_{0}\left(\bmod p_{i}\right)\right\} \leq 2 s_{1}-1 .
$$

Hence, for $1 \leq i \leq r$ and for any $h^{\prime} \in \mathbb{Z}_{p_{i}}, \mathbf{h}_{v} \in \mathbb{Z}^{k}(v=0, \ldots, s-1)$ with $\left(\mathbf{h}_{0}, \ldots, \mathbf{h}_{s-1}\right) \not \equiv \mathbf{0}\left(\bmod p_{i}\right)$,

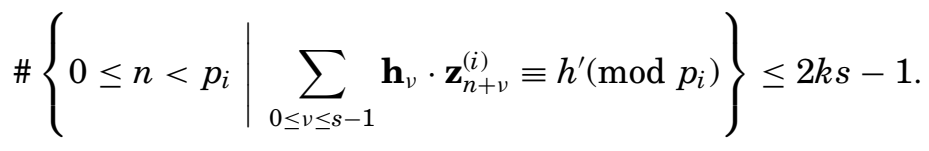

Therefore, Theorem $1^{\prime}$ can be applied with $B_{i} \leq 2 k s-1$.

Bearing in mind that for $p \geq 5$ and $B \in[0,2(p-1)]$,

$$
\frac{\sqrt{p B}+1}{\sqrt{p-1}} \leq \sqrt{B+2}+0.5
$$

we get the assertion of Theorem 4 . 


\subsection{The Compound Explicit Inversive Method}

The explicit inversive congruential method was introduced in EichenauerHerrmann [1993b]. The compound version of this method was studied in Eichenauer-Herrmann [1993a]. The following $k$-dimensional variant of the explicit inversive congruential method was proposed in Niederreiter [1994]. For the average-case analysis, see Eichenauer-Herrmann et al. [1997].

For $1 \leq i \leq r$, let $a_{i 1}, \ldots, a_{i k} \in \mathbb{Z}_{p_{i}}^{*}$ and $b_{i 1}, \ldots, b_{i k} \in \mathbb{Z}_{p_{i}}$ be arbitrary fixed parameters. Let $z_{n j}^{(i)} \in \mathbb{Z}_{p_{i}}$ be defined by

$$
z_{n j}^{(i)} \equiv\left(a_{i j} n+b_{i j}\right)^{-1}\left(\bmod p_{i}\right), \quad n \geq 0, \quad 1 \leq j \leq k,
$$

where $z^{-1}$ denotes the multiplicative inverse of $z \in \mathbb{Z}_{p_{i}}^{*}$ and $0^{-1}=0$. It is obvious that the sequence $\left(\mathbf{z}_{n}^{(i)}\right)_{n \geq 0}$ of elements of $\mathbb{Z}_{p_{i}}^{k}$ given by

$$
\mathbf{z}_{n}^{(i)}=\left(z_{n 1}^{(i)}, \ldots, z_{n k}^{(i)}\right), \quad n \geq 0,
$$

is purely periodic, with period length $p_{i}$. Now, in the compound approach, the corresponding stream of $k$-dimensional vectors of (ordinary) explicit inversive congruential pseudorandom numbers is defined by

$$
\mathbf{x}_{n}^{(i)} \equiv \frac{c_{i} \mathbf{z}_{n}^{(i)}}{p_{i}}(\bmod 1), \quad n \geq 0,
$$

where $c_{i} \in \mathbb{Z}_{p_{i}}^{*}$ is an additional parameter.

Using Theorem $1^{\prime}$, we obtain the following discrepancy estimate for the distribution of $k s$-dimensional sequence $\left(\mathbf{x}_{n}, \ldots, \mathbf{x}_{n+s-1}\right) \in[0,1)^{k s}$ :

Theorem 5. Let $1 \leq k s \leq \min \left\{p_{1}, \ldots, p_{r}\right\}$. For $1 \leq i \leq r$, let $\left(a_{i j}^{-1} b_{i j}+v\right) \in \mathbb{Z}_{p_{i}}$ $(j=1, \ldots, k, v=0, \ldots, s-1)$ be distinct elements and $\varepsilon \in(0,1]$. Then there are more than $(1-\varepsilon)\left(p_{1}-1\right) \cdots\left(p_{r}-1\right)$ values of $\left(c_{1}, \ldots, c_{r}\right) \in \mathbb{Z}_{p_{1}}^{*} \times \cdots \times \mathbb{Z}_{p_{r}}^{*}$ with

$$
D_{N ; c_{1}, \ldots, c_{r}}^{(k s)}<\left(\frac{3}{2}\right)^{k s} \frac{3}{\varepsilon} \prod_{i=1}^{r} \frac{\sqrt{2 k s p_{i}}+1}{\sqrt{p_{i}-1}} N^{-1 / 2}(2 \log N+4)^{k s+2} \log ^{3 / 2}\left(\log _{2} N+4\right)
$$

for all $N \in[1, m]$.

Proof. $\quad$ For $1 \leq i \leq r, 1 \leq s_{1}<p_{i}, h_{0} \in \mathbb{Z}_{p_{i}}$, and $\mathbf{h} \in \mathbb{Z}^{s_{1}}, \mathbf{h} \not \mathbf{0}\left(\bmod p_{i}\right)$, it follows from Niederreiter [1994, Theorem 1] and Eichenauer-Herrmann et al. [1997, p. 215], that

$$
\#\left\{0 \leq n<p_{i} \mid \mathbf{h} \mathbf{z}_{n}^{(i)} \equiv h_{0}\left(\bmod p_{i}\right)\right\} \leq 2 s_{1} .
$$

Hence, for $1 \leq i \leq r$ and for any $h^{\prime} \in \mathbb{Z}_{p_{i}}, \mathbf{h}_{v} \in \mathbb{Z}^{k}(v=0, \ldots, s-1)$ with $\left(\mathbf{h}_{0}, \ldots, \mathbf{h}_{s-1}\right) \not \equiv \mathbf{0}\left(\bmod p_{i}\right)$,

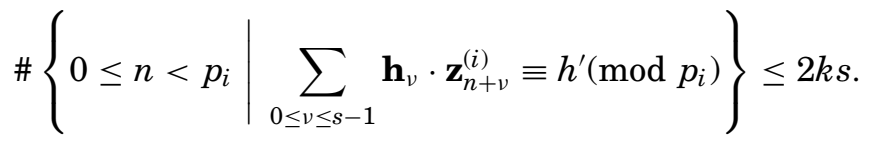

Therefore, Theorem $1^{\prime}$ can be applied with $B_{i} \leq 2 k s$, which yields the desired result. 
Remark 2. In Levin [2000], similar results are obtained for the digital inversive pseudorandom number generators [Eichenauer-Herrmann and Niederreiter 1994].

Remark 3. In accordance with the law of the iterated logarithm for the discrepancy of $N$ true random points $\mathbf{z}_{n} \in[0,1)^{d}(n=1, \ldots, N)$ [Kiefer 1961],

$$
D\left(\mathbf{z}_{1}, \ldots, \mathbf{z}_{N}\right)=O\left(N^{-1 / 2}(\log \log N)^{1 / 2}\right) \quad \lambda \text { a.e. }
$$

It is obvious that for large $d$, the upper bounds (16) are very far from the order of magnitude (32). Using the generators of pseudorandom numbers based on low discrepancy sequences [Levin 1999], we obtain a discrepancy estimate with an order of magnitude (32) for the large $d$.

\section{CONCLUSION}

Let the number of prime factors $r$ be fixed. Then Theorem $1^{\prime}$ shows that there exist parameters $\left(c_{1}, \ldots, c_{r}\right)$ such that the discrepancy $D_{N ; c_{1}, \ldots, c_{r}}^{(d)}$ over part of the period of $d$-tuples of successive pseudorandom numbers is of order of magnitude $O\left(N^{-1 / 2}(\log N)^{d+3}\right)$ for all $N \in[1, m]$. This fits well with the asymptotic behavior of the discrepancy of $N$ true random points from $[0,1)^{d}$ according to the law of the iterated logarithm, provided that the degree $d$ is not large. In Section 3.2, we propose the heuristic algorithm for the choice of such parameters $\left(c_{1}, \ldots, c_{r}\right) \in \mathbb{Z}_{p_{1}}^{*} \times \cdots \times \mathbb{Z}_{p_{r}}^{*}$ for not too large $d$ and $m$ (say $d \leq 5, m \leq 10^{9}$ ). The considered pseudorandom numbers can be used also in simulation methods for the case when the number of calculations is not known in advance.

Theorem 2 is the analog of Theorem $1^{\prime}$, where we use the diaphony instead of the discrepancy to characterize the uniformity and statistical independence of the successive pseudorandom numbers.

Theorem 3, 4, and 5 are immediate consequences of Theorem $1^{\prime}$ for the compound nonlinear, inversive and explicit inversive congruential method.

\section{ACKNOWLEDGMENT}

The author would like to thank Pierre L'Ecuyer and Harald Niederreiter for many corrections and suggestions that improved this paper.

\section{REFERENCES}

Cochrane, T. 1988. Trigonometric approximation and uniform distribution modulo one. Proc. AMS 103, 695-702.

Drmota, M., And Tichy, R. F. 1997. Sequences, Discrepancies and Applications, Lecture Notes in Mathematics, vol. 1651. Springer-Verlag, New York.

Eichenauer, J., Grothe, H., AND LeHN, J. 1988. Marsaglia's lattice test and non-linear congruential pseudorandom number generators. Metrika 35, 241-250.

Eichenauer, J., AND LeHn, J. 1986. A non-linear congruential pseudorandom number generator. Statist. Papers 27, 315-326.

Eichenauer-Herrmann, J. 1993a. Explicit inversive congruential pseudorandom numbers: The compound approach. Computing 51, 175-182.

EichenaUER-HERrmanN, J. 1993b. Statistical independence of a new class of inversive congruential pseudorandom numbers. Math. Comp. 60, 375-384.

ACM Transactions on Modeling and Computer Simulation, Vol. 11, No. 3, July 2001. 
Eichenauer-Herrmann, J. 1994a. Compound nonlinear congruential pseudorandom numbers. Monatsh. Math. 117, 213-222.

Eichenauer-HerRmann, J. 1994b. On generalized inversive congruential pseudorandom numbers. Math. Comput. 63, 293-299.

Eichenauer-Herrmann, J. 1995. Pseudorandom number generation by nonlinear methods. Internat. Statist. Rev. 63, 247-255.

Eichenauer-Herrmann, J., AND Emmerich, F. 1996. Compound inversive congruential pseudorandom numbers: An average case analysis. Math. Comput. 65, 215-225.

Eichenauer-HerRmann, J., Emmerich, F., and Larcher, G. 1997. Average discrepancy, hyperplanes, and compound pseudorandom numbers. Finite Fields Appl. 3, 203-218.

EichenaUtR-HERRMANN, J., AND LARCHER, G. 1996. Average behaviour of compound nonlinear congruential pseudorandom numbers. Finite Fields Appl. 2, 111-123.

Eichenauer-Herrmann, J., AND Niederreiter, H. 1994. Digital inversive pseudorandom numbers. ACM Trans. Model. Comput. Simulation 4, 339-349.

Eichenauer-Herrmann, J., ANd Niederreiter, H. 1997. Parallel streams of nonlinear congruential pseudorandom numbers. Finite Fields Appl. 3, 219-233.

HeINRICH, S. 1996. Efficient algorithms for computing $L_{2}$ discrepancy. Math. Comput, 65, 216, 1621-1633.

Hellekalek, P., AND NiederReiter, H. 1998. The weighted spectral test: diaphony. ACM Trans. Model. Comput. 8, 43-60.

KIEFER, J. 1961. On large deviations of the empiric d.f. of vector chance variables and a law of the iterated logarithm. Pacific J. Math. 11, 649-660.

KnUth, D. 1981. The Art of Computer Programming, Volume 2. Addison-Wesley, San Francisco, Calif.

Kововоv, N. M. 1992. Exponential Sums and Their Applications. Kluwer Academic Publishers, Dordrecht, Germany.

L'ECUyer, P. 1994. Uniform random number generation. Ann. Oper. Res. 53, 77-120.

Lev, V. F. 1995. $L_{2}$-discrepancy and geometrical interpretation of diaphony. Acta Math. Hungar. 69, 281-300.

LeVIN, M. B. 1989. On the choice of parameters in generators of pseudorandom numbers. Sov. Math. Dokl. 40, 101-105.

Levin, M. B. 1999. Discrepancy estimates of completely uniform distributed sequences and pseudorandom sequence. Intern. Math. Res. Notes 22, 1231-1251.

Levin, M. B. 2000. Explicit digital inversive pseudorandom numbers. Math. Slovaca., 50, 5, 581-598.

NiederReiter, H. 1977. Pseudo-random numbers and optimal coefficients. Adv. Math. 26,99-181.

NiederReiter, H. 1978. Quasi-monte carlo methods and pseudorandom numbers. Bull. Amer. Math. Soc. 84, 957-1041.

NiederReiter, H. 1992. Random Number Generation and Quasi-Monte Carlo Methods. SIAM, Philadelphia, Pa.

NiEDERREITER, H. 1994. On a new class of pseudorandom numbers for simulation methods. J. Comput. Appl. Math. 56, 159-167.

StegBuchner, H. 1979. Eine mehrdimensionale version der ungleichung von leveque. Monatsh. Math. 87, 167-169.

ZINTERHOF, P. 1976. Über einige abschätzungen bei der approximation von funktionen mit gleichverteilungsmethoden. Ósterr. Akad. Wiss. SB II 185, 121-132.

Received August 1998; revised October 2000; accepted November 2000 